\title{
Original
} Article

\section{Mid-Term Outcomes of Acute Type B Aortic Dissection in Japan Single Center}

\author{
Tomoyuki Minami, MD, ${ }^{1}$ Kiyotaka Imoto, MD, ${ }^{1}$ Keiji Uchida, MD, ${ }^{1}$ Shota Yasuda, MD, ${ }^{1}$ \\ Norihisa Karube, MD, ${ }^{1}$ Shinichi Suzuki, MD, ${ }^{2}$ and Munetaka Masuda, $\mathrm{MD}^{2}$
}

\begin{abstract}
Purpose: To study mid-term outcomes in patients admitted to receive treatment for acute type B aortic dissection.

Methods: The study group comprised 229 patients with acute type B aortic dissection treated between January 2000 and July 2010.128 patients had a thrombosed false lumen, and 101 had a patent false lumen.

Results: In the thrombosed group, 6 had rupture, 4 had malperfusion, and 118 had no complications. There were 5 early deaths (3.9\%). In the patent group, 12 had rupture, 19 had malperfusion, and 70 had no complications. There were 6 early deaths $(5.9 \%)$. Overall survival rates in the thrombosed group and the patent group were $94.7 \pm 2.2 \%$ and $90.2 \pm 3.2 \%$ at 1 year, and $84.3 \pm 4.6 \%$ and $85.9 \pm 4.3 \%$ at 5 years $(p=0.892)$, respectively. Aorta-related event-free rates were $85.6 \pm 3.4 \%$ and $48.3 \pm 5.5 \%$ at 1 year, and $76.0 \pm 5.1 \%$ and $35.2 \pm 7.2 \%$ at 5 years $(p<0.001)$, respectively.

Conclusions: The incidences of rupture and malperfusion during the acute phase were higher in the patent group compared with the thrombosed group. At the late period, although the aorta-related event rate was higher in the patent group, the survival rate did not differ between two groups. Close follow-up and aggressive intervention strategy of the patent group may result comparable outcomes with the thrombosed group.
\end{abstract}

Keywords: aortic dissection, stanford type B, false lumen, TEVAR, open surgical repair

\section{Introduction}

Acute aortic dissection is a serious cardiovascular condition, and surgery is the mainstay of treatment for acute type A aortic dissection because of its high mortality with conservative treatment. ${ }^{1,2)}$ Acute type B aortic

${ }^{1}$ Yokohama City University Medical Center, Cardiovascular Center, Yokohama, Kanagawa, Japan

${ }^{2}$ Department of Surgery, Yokohama City University Hospital, Yokohama, Kanagawa, Japan

Received: June 20, 2012; Accepted: June 26, 2012 Corresponding author: Tomoyuki Minami, MD. Yokohama Municipal Citizen's Hospital, Cardiovascular surgery, Okazawa-cho 56, Hodogaya-ku, Yokohama, Kanagawa 240-8555, Japan

Email: tomoyuki3711@train.ocn.ne.jp

(C)2013 The Editorial Committee of Annals of Thoracic and Cardiovascular Surgery. All rights reserved. dissection is usually managed by conservative treatment with antihypertensive agents and rest. However, patients with acute type B aortic dissection who have complications such as rupture and organ ischemia have high mortality; such patients thus often require emergency surgery. ${ }^{3-6)}$ Late complications such as aortic enlargement and rupture may occur during follow-up in patients who receive conservative therapy at the acute phase, thereby necessitating surgery. ${ }^{7-9)}$

Several studies have reported that early or late complications such as aortic rupture and organ ischemia are related to whether the false lumen has been thrombosed or remains patent. ${ }^{10,11)}$ Akutsu, et al. reported that a patent false lumen is strongly associated with late aortic events and mortality. ${ }^{12)}$ However there are few reports focusing on the false lumen of acute type B dissection in relation to early and late outcomes. 
We retrospectively studied patients with acute type B aortic dissection who were hospitalized and treated in our institution. The aim of this study was to clarify the impact of complete occlusion of false lumen at the time of presentation on early and late outcomes.

\section{Materials and Methods}

\section{Study population}

The study group comprised 229 consecutive patients with acute type B aortic dissection who were admitted to Yokohama City University Medical Center from January 2000 through July 2010. Computed tomography (CT) was performed in our hospital or by a local physician at the time of symptom onset. The diagnosis was established by confirming the presence of a false lumen of the aorta on CT performed within 14 days after the onset of chest and/or back pain. Dissection due to iatrogenic reasons was excluded from the study.

The 229 patients (155 men, 74 women) were divided into 2 groups according to the status of the false lumen on CT images: 128 with complete thrombotic occlusion of the false lumen (thrombosed group) and 101 with patent false lumen (patent group). The mean age of patients was $66.6 \pm 12.2$ years, and the cumulative follow-up period was 657.5 patient-years. The complete follow up rate was $81.8 \%$.

\section{Patient characteristics}

Disease history was ascertained on the basis of personal interviews with the patient at admission. The maximum diameter of the aorta was measured on CT scans.

\section{Medical therapy}

All patients who were admitted for acute type B aortic dissection received antihypertensive therapy. In accordance with the treatment protocol of our hospital, a calcium antagonist was given as a continuous intravenous infusion to maintain a target systolic blood pressure of $130 \mathrm{~mm} \mathrm{Hg}$, and nitroglycerin preparations were additionally administered as required. Oral antihypertensive therapy was begun 2 days after the onset of symptom with beta-blockers, angiotensin-receptor blockers, and/or calcium-channel blockers, as required. Pain was adequately controlled by treatment with non-steroidal anti-inflammatory drugs; if necessary, narcotics was used. CT was performed around 7 and 18 days after the onset of acute type B aortic dissection to confirm the status of the false lumen and the increase of aortic diameter.
Patients without major events during hospitalization were discharged around 21 days after the admission.

\section{Surgical therapy}

Surgery was indicated in addition to medical therapy for patients who had rupture, organ ischemia, or both. Rupture and organ ischemia were diagnosed on the basis of CT and physical examination during the acute phase. Rupture was basically treated by open surgery or by thoracic endovascular aortic repair (TEVAR). Choice of treatment strategy was determined by anatomical reasons and availability of clinical team. Organ ischemia was treated by bypass surgery or catheter intervention. If patients who had rupture or organ ischemia refused surgical interventions, they were managed by conservative treatment.

\section{Factors influencing mid-term outcomes}

Follow-up data were obtained from the patients' medical records or by telephone call. Patients whose survival status was unknown at July 2010 were considered lost from follow-up. Late aortic events were defined as aortic surgery, progression to acute type A aortic dissection, recurrence of acute type $\mathrm{B}$ aortic dissection, and rupture.

\section{Statistical analysis}

The data are expressed as means \pm standard deviation of the mean. Un-paired student's t-test was used to compare continuous variables, and chi-square test and Fisher's exact test were used to compare categorical variables. Time-related changes in freedom from aortarelated events and survival were analyzed by the KaplanMeier method and Mantel-Cox proportional hazards model. Statistical analysis was made by using SPSS software (version 18 for Windows, IBM Inc.). P value equal or less than 0.05 was considered statistically significant.

\section{Results}

\section{Patient demographic characteristics}

Table 1 shows the baseline clinical characteristics of the 128 patients in the thrombosed group and the 101 patients in the patent group. Mean age was significantly higher in the thrombosed group $(68.9 \pm 10.1$ years) than that in the patent group $(63.7 \pm 14.0$ years $)$. The gender did not differ significantly between the groups. There were no differences between the groups in the previous history of diseases such as hypertension, hyperlipidemia, diabetes mellitus, chronic obstructive pulmonary disease, 
Table 1 Baseline clinical characteristics

\begin{tabular}{lccc}
\hline & $\begin{array}{c}\text { Thrombosed } \\
\mathrm{n}=128\end{array}$ & $\begin{array}{c}\text { Patent } \\
\mathrm{n}=101\end{array}$ & $\mathrm{p}$ \\
\hline Age & $68.9 \pm 10.1$ & $63.7 \pm 14.0$ & 0.001 \\
Gender (m/f) & $82 / 46$ & $73 / 28$ & 0.187 \\
Hypertension & $90(70.3 \%)$ & $69(68.3 \%)$ & 0.754 \\
Hyperlipidemia & $17(13.3 \%)$ & $12(11.9 \%)$ & 0.752 \\
Diabetes & $7(5.5 \%)$ & $5(5.0 \%)$ & 0.861 \\
COPD & $6(4.7 \%)$ & $3(3.0 \%)$ & 0.507 \\
Previous cardiac surgery & $10(7.8 \%)$ & $12(11.9 \%)$ & 0.3 \\
Maximum diameter of aorta & 38.4 & 40.7 & 0.352 \\
DeBakey IIIa & $72(56.3 \%)$ & $10(9.9 \%)$ & $<0.001$ \\
DeBakey IIIb & $56(43.7 \%)$ & $91(90.1 \%)$ & \\
\hline
\end{tabular}

COPD: chronic obstructive pulmonary disease

Table 2 Complications in acute phase

\begin{tabular}{lccc}
\hline & $\begin{array}{c}\text { Thrombosed } \\
\mathrm{n}=128\end{array}$ & $\begin{array}{c}\text { Patent } \\
\mathrm{n}=101\end{array}$ & $\mathrm{p}$ \\
\hline No complications & $118(92.2 \%)$ & $70(69.3 \%)$ & $<0.001$ \\
Rupture & $6(4.7 \%)$ & $12(11.9 \%)$ & 0.045 \\
Malperfusion-spinal & $0(0 \%)$ & $2(2.0 \%)$ & 0.11 \\
Mesenteric & $2(1.6 \%)$ & $4(4.0 \%)$ & 0.259 \\
Renal & $0(0 \%)$ & $3(3.0 \%)$ & 0.05 \\
Lower limb & $2(1.6 \%)$ & $10(9.9 \%)$ & 0.005 \\
Surgery in acute phase & $7(5.4 \%)$ & $26(25.7 \%)$ & 0.001 \\
\hline
\end{tabular}

or previous cardiac surgery. The maximum aortic diameter at disease onset was also similar. DeBakey classification was significantly different between the groups. Extension of aortic dissection to the abdominal aorta was seen more frequently in the patent group than in the thrombosed group (Table 1).

\section{Complications in the acute phase}

Complications occurring during the acute phase are shown in Table 2. In the thrombosed group, 6 patients had rupture, and 4 had organ ischemia (mesenteric ischemia in 2 and lower limb ischemia in 2). The other 118 patients had no major complications. In the patent group, 12 patients had rupture, and 19 had organ ischemia (spinal ischemia in 2, mesenteric ischemia in 4 , renal ischemia in 3, and lower limb ischemia in 10). The other 70 patients had no major complications. The number of patients who received surgery during the acute phase of aortic dissection was $7(5.4 \%)$ in the thrombosed group (rupture in 3, mesenteric ischemia in 2, and lower extremity ischemia in 2), and $26(25.7 \%)$ in the patent group (rupture in 10, mesenteric ischemia in 3, renal ischemia in 2, and lower extremity ischemia in 2). The proportion of patients who had acute type B aortic dissection without complications was significantly higher in the thrombosed group. The incidence of rupture, lower limb ischemia and surgery during the acute phase were significantly higher in the patent group (Table 2).

\section{Early outcomes}

In the thrombosed group, 5 patients (3.9\%) died within 30 days after admission. The cause of death was rupture in 2 patients, mesenteric ischemia in 2, and respiratory failure in 1 . In the patent group, $6(5.9 \%)$ died within 30 days after admission. The cause of death was rupture in 2 patients and mesenteric ischemia in 4 . The early mortality rate did not differ between the thrombosed group and the patent group. In this series, all patients with mesenteric ischemia died in the early period.

\section{Treatment for complications in the early phase}

In the thrombosed group, 6 patients had rupture in the early phase. Three of these patients underwent open surgery. The other 3 patients were observed: 2 were not underwent surgery because of their refusal (1 died during hospitalization, and 1 was transferred to another hospital 
Table 3 Details of late aortic events

\begin{tabular}{lll}
\hline & $\begin{array}{c}\text { Thrombosed } \\
\mathrm{n}=123\end{array}$ & $\begin{array}{c}\text { Patent } \\
\mathrm{n}=95\end{array}$ \\
\hline Elective aortic surgery & $7(4.8 \%)$ & $15(15.8 \%)$ \\
Open surgery for dilatation & 3 & 6 \\
TEVER for dilatation & 4 & 1 \\
TEVER for entry closure & 0 & 8 \\
Development of type A & $1(0.8 \%)$ & $3(3.2 \%)$ \\
Recurrence of type B & $3(3.3 \%)$ & $3(3.2 \%)$ \\
Rupture & $3(2.4 \%)$ & $1(1.1 \%)$ \\
\hline
\end{tabular}

TEVAR: thoracic endovascular aortic repair

on 42 day after the onset) and 1 had shock on admission and died subsequently. In the patent group, 12 patients had rupture, 10 of them underwent surgery. Open surgery was performed in 9 patients, 2 of them died intraoperatively. TEVAR with a custom-made stent graft was performed in 1 patient. The other 2 patients with rupture received conservative therapy and were transferred to another hospital 14 and 21 days after the onset of symptom.

In the thrombosed group, intervention for organ ischemia was performed in 2 patients with mesenteric ischemia and 2 with lower limb ischemia. One of the patients with mesenteric ischemia underwent total small-bowel resection, and the other underwent superior mesenteric artery bypass surgery. Both patients died during hospitalization. The 2 patients with lower limb ischemia underwent extra-anatomical bypass surgery and were discharged from the hospital in good condition.

In the patent group, 2 patients had spinal ischemia, 4 had mesenteric ischemia, 3 had renal ischemia, and 10 had lower limb ischemia. The 2 patients with spinal ischemia had paraplegia at the time of discharge, although one patient with underwent spinal drainage. Laparotomy was done in 3 of 4 patients with mesenteric ischemia. The other patient refused surgery and received conservative therapy. All 4 patients with mesenteric ischemia died during hospitalization. One patient with renal ischemia received conservative therapy, and the other 2 underwent catheter intervention. Introduction of dialysis was avoided in all 3 patients. Of 10 patients with lower limb ischemia, extra-anatomical bypass surgery was performed in 8, TEVAR for entry closure in 1, and catheter fenestration in 1 . All 10 patients were discharged in good condition.

\section{Aortic events at the late phase}

In the thrombosed group, 14 patients had late aortic events. Seven of these patients underwent elective aortic
Table 4 Causes of late death

\begin{tabular}{lcc}
\hline & Thrombosed & Patent \\
\hline Operative death & 0 & 0 \\
Development of type A & 1 & 0 \\
Rupture & 2 & 1 \\
Chronic heart failure & 1 & 0 \\
Acute myocardial infarction & 1 & 0 \\
Cancer & 3 & 1 \\
Other & 4 & 2 \\
\hline
\end{tabular}

surgery during follow-up. Acute type A aortic dissection developed in 1 patient who died without surgery. Acute type B aortic dissection recurred in 3 patients, all of them received conservative therapy. Rupture occurred in 3 patients, and 2 of them died without surgical intervention (Table 3 ).

In the patent group, 22 patients had late aortic events. Fifteen of them underwent elective aortic surgery. Acute type A aortic dissection developed in 3 patients, and all of them underwent surgery. Acute type B aortic dissection recurred in 3 patients, and all of them received conservative therapy. Rupture occurred in 1 patient, and he was treated with conservative therapy (Table 3).

\section{Late mortality}

No patient died after elective aortic surgery. In the thrombosed group, 1 patient died due to acute type A aortic dissection, and 2 patients died due to rupture. In the patent false lumen group, 1 patient died due to rupture (Table 4).

\section{Treatments for events at the late phase}

In all 7 patients who underwent elective aortic surgery in the thrombosed group because of the increase of the diameter of the aorta. Three patients underwent open surgery, and the other 4 patients underwent TEVAR. An endoleak developed in 1 who underwent TEVAR. This patient subsequently underwent open surgery for expansion of the aorta. There was no surgery-related death.

Of the 15 patients who underwent elective aortic surgery in the patent group, 6 underwent thoracotomy or laparotomy for aortic expansion (including the abdominal aorta), and 9 underwent TEVAR. Indication for TEVAR was aortic dilatation in 1 and closure of entry in 8 . Among the patients underwent TEVAR, false lumen was fully thrombosed immediately after surgery in 2 , and endoleak was developed in 6 . The endoleak disappeared during follow-up in 3 patients. The other two patients 

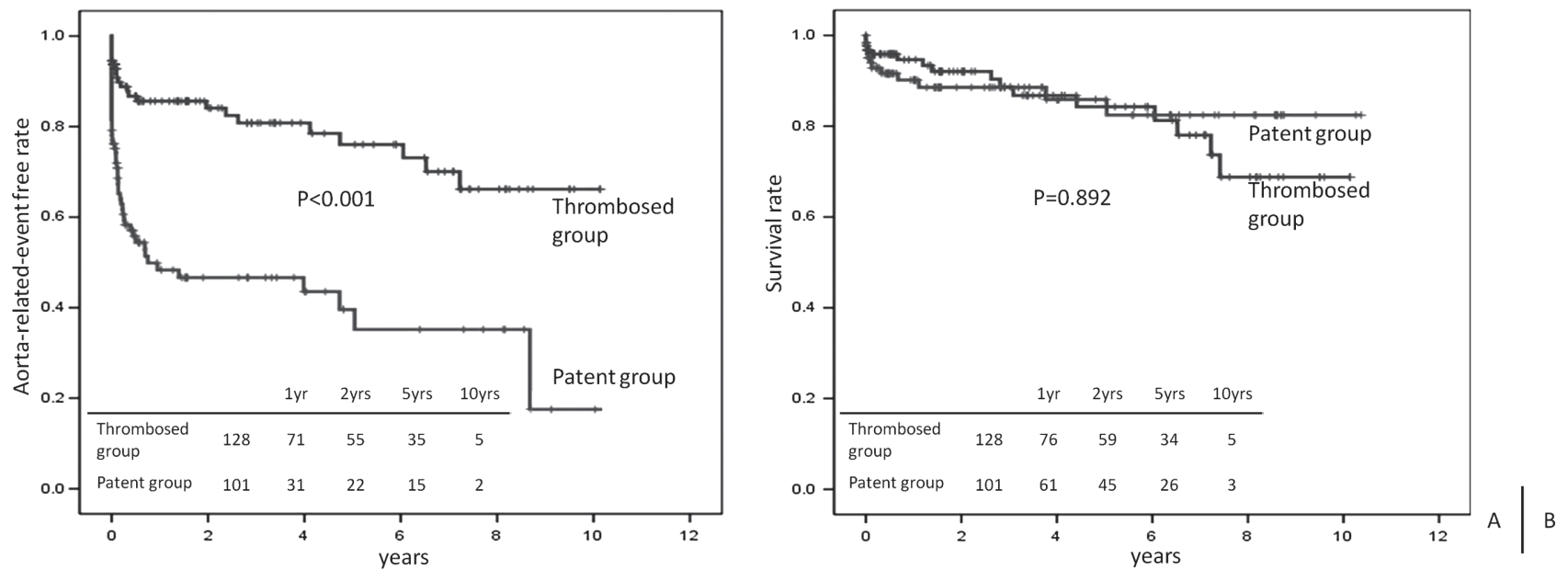

Fig. 1 Aorta-related-event-free rate (A) and survival rate (B) of patients in the thrombosed group and the patent group. The incidence of aorta-related-event was significantly higher in the patent group than in the thrombosed group $(\mathrm{p}<0.001)$. In contrast, a significant difference between the two groups was not found in terms of death from any causes.

underwent elective open surgery because of aortic expansion, and the remaining 1 patient is under observation at the outpatient clinic (Table 3).

\section{Aorta-related-event-free rate}

In the thrombosed group, the aorta-related-event-free rate was $85.6 \% \pm 3.4 \%$ at 1 year, and $76.0 \% \pm 5.1 \%$ at 5 years. In the patent group, the aorta-related-event-free rate was $48.3 \% \pm 5.5 \%$ at 1 year, and $35.2 \% \pm 7.2 \%$ at 5 years. The difference between the groups was significant $(\mathrm{p}<0.001)$ (Fig. 1A).

\section{Mid-term survival}

In the thrombosed group, the survival rate was $94.7 \% \pm 2.2 \%$ at 1 year, and $84.3 \% \pm 4.6 \%$ at 5 years. In the patent group, the survival rate was $90.2 \% \pm 3.2 \%$ at 1 year, and $85.9 \% \pm 4.3 \%$ at 5 years. The difference between the groups was not significant $(\mathrm{p}=0.892)$ (Fig. 1B).

\section{Discussion}

The objective of this study was to clarify the impact of thrombotic occlusion of the false lumen at the time of presentation in acute type B aortic dissection on early and late outcomes. During the acute phase, the incidence of complications such as rupture and organ ischemia was significantly higher in the patent group than in the thrombosed group. Even in the late phase, the incidence of aortic events was significantly higher in the patent group than in the thrombosed group. Several studies have reported that patent false lumen is an important prognostic factor in patients with aortic dissection. ${ }^{10-12)}$

Early death occurred in 5 patients $(3.9 \%)$ in the thrombosed group and $6(5.9 \%)$ in the patent group. This difference was not significant. In the thrombosed group, surgery was not performed in 3 patients with rupture because of their refusal for surgery, and 2 of these patients died during hospitalization. This may have resulted increased mortality rate in the thrombosed group in our series. All patients with mesenteric ischemia died, irrespective of the status of false lumen, and this is one of the most serious problems to be solved.

Metha, et al. reported that good outcome was obtained with medical treatment in patients with acute type B aortic dissection without complications with an early mortality rate of only about $1 \% .^{13)}$ While high in-hospital mortality rate of $29 \%$ was reported in case of surgical intervention due to complications, and the mortality rate goes up higher than $50 \%$ in case of emergent operation. ${ }^{14,15)}$ In general, open surgery is indicated for the patients with uncontrollable back pain, rapidly expanding false lumen, rupture, or ischemia of main organs when catheter intervention is not feasible. ${ }^{16-18)}$

In our hospital, Open surgery was performed only in patients who had rupture at the time of presentation ( 3 in the thrombosed group and 9 in the patent group). The surgical procedures were aortic arch replacement in 5 patients, replacement of the descending aorta in 6 , and replacement of the thoraco-abdominal aorta in 1. Two of these patients (16.7\%) died intraoperatively. Recently, thoracic endovascular aortic repair (TEVAR) has been 
developed to treat acute type B aortic dissection associated with complications with satisfactory outcomes. ${ }^{19-21)}$ Steuer, et al. reported the 30-day mortality rate of 3\% and 5-year survival rate of $87 \%$ with TEVAR. ${ }^{20)}$ Since 2010, we have introduced aggressive stent grafting strategy for the patients with rupture.

As for treatment strategies for organ ischemia, bypass surgery and catheter intervention are our choice of treatment. There were no deaths among patients with lower limb ischemia and renal ischemia in our series. Bypass surgery and catheter intervention are thus considered appropriate treatments for lower limb ischemia and renal ischemia. On the other hand, all patients with mesenteric ischemia died. Mesenteric ischemia caused by acute aortic dissection is still a lethal complication to be solved. Mesenteric ischemia leads to irreversible changes within several minutes, triggering mesenteric necrosis and acidosis, resulting in death. The patency of the superior mesenteric artery should be confirmed on the diagnosis of aortic dissection, and early intervention or central repair should be performed as soon as possible when mesenteric ischemia is suspected.

As for late outcomes, the incidence of aortic events was significantly higher in the patent group than in the thrombosed group. Although TEVAR was performed to close the entry site in case of patent false lumen, usefulness of TEVAR in patients with acute type B aortic dissection without complications remains unclear. Some studies mentioned that stent grafting promotes false lumen thrombosis and remodeling of major blood vessels. ${ }^{22,23)}$ If the future risk of aortic events depends on the status of the false lumen, thrombotic occlusion of the false lumen may improve long-term outcomes. Because TEVAR is considered ineffective in patients with an expanded false lumen, we believe that entry closure by TEVAR should be performed relatively early phase before the expansion of false lumen.

The survival rate did not differ significantly between the thrombosed group and the patent group. Significantly higher age of the thrombosed group may contribute this result. In addition, our strategy of aggressive intervention for acute type B aortic dissection with patent false lumen might have improved the survival rate in these patients. In patients with patent false lumen, TEVAR is recommended when closure of entry site is possible. When the aortic diameter increases during follow-up, open surgery is recommended. In patients with thrombosed false lumen, both TEVAR and open surgery is recommended when the aortic diameter increases.
Our study had several limitations. First, our study is not prospective and our results may have been biased by the presence of censored patients and the relatively small sample size. Second, we did not analyze factors such as length of the initial hospital stay, control of blood pressure in the outpatient clinic, and aortic diameter before the onset of aortic dissection.

In conclusion, the incidences of rupture and organ ischemia were higher in the patent group than in the thrombosed group in the acute phase. In the late phase, the incidence of aortic events was higher in the patent group, however, there was no difference in the survival rate as compared with the thrombosed group. These results suggest that intensive follow-up of acute type B aortic dissection patients with patent false lumen and our aggressive intervention strategy might improve the longterm survival in these patients.

\section{Disclosure Statement}

The authors declare no conflicts of interest.

\section{References}

1) Suzuki S, Masuda M. An update on surgery for acute type A aortic dissection: Aortic root repair, endovascular stent graft, and genetic research. Surg Today 2009; 39: 281-9.

2) Goda M, Imoto K, Suzuki S, et al. Risk analysis for hospital mortality in patients with acute type a aortic dissection. Ann Thorac Surg 2010; 90: 1246-50.

3) Daily PO, Trueblood HW, Stinson EB, et al. Management of acute aortic dissections. Ann Thorac Surg 1970; 10: $237-47$.

4) Elefteriades JA, Lovoulos CJ, Coady MA, et al. Management of descending aortic dissection. Ann Thorac Surg 1999; 67: 2002-5; discussion 2014-19.

5) Anagnostopoulos CE, Prabhakar MJ, Kittle CF. Aortic dissections and dissecting aneurysms. Am J Cardiol 1972; 30: 263-73.

6) Crawford ES, Svensson LG, Coselli JS, et al. Aortic dissection and dissecting aortic aneurysms. Ann Surg 1988; 208: 254-73.

7) Juvonen T, Ergin MA, Galla JD, et al. Risk factors for rupture of chronic type $\mathrm{B}$ dissections. J Thorac Cardiovasc Surg 1999; 117: 776-86.

8) Masuda Y, Yamada Z, Morooka N, et al. Prognosis of patients with medically treated aortic dissections. Circulation 1991; 84: III7-13.

9) Tsai TT, Fattori R, Trimarchi S, et al. Long-term survival in patients presenting with type B acute aortic dissection: Insights from the International Registry of Acute Aortic Dissection. Circulation 2006; 114: 2226-31. 
10) Shimizu H, Yoshino H, Udagawa $H$, et al. Prognosis of aortic intramural hemorrhage compared with classic aortic dissection. Am J Cardiol 2000; 85: 792-5, A10.

11) Marui A, Mochizuki T, Mitsui N, et al. Toward the best treatment for uncomplicated patients with type B acute aortic dissection: A consideration for sound surgical indication. Circulation 1999; 100: II275-80.

12) Akutsu K, Nejima J, Kiuchi K, et al. Effects of the patent false lumen on the long-term outcome of type $\mathrm{B}$ acute aortic dissection. Eur J Cardiothorac Surg 2004; 26: 359-66.

13) Mehta RH, Bossone E, Evangelista A, et al. Acute type B aortic dissection in elderly patients: Clinical features, outcomes, and simple risk stratification rule. Ann Thorac Surg 2004; 77: 1622-8; discussion 1629.

14) Bozinovski J, Coselli JS. Outcomes and survival in surgical treatment of descending thoracic aorta with acute dissection. Ann Thorac Surg 2008; 85: 965-70; discussion 970-1.

15) Umaña JP, Miller DC, Mitchell RS. What is the best treatment for patients with acute type B aortic dissections-medical, surgical, or endovascular stentgrafting? Ann Thorac Surg 2002; 74: S1840-3; discussion S1857-63.

16) Lansman SL, Hagel C, Fink D, et al. Acute type B aortic dissection: Surgical therapy. Ann Thorac Surg. 2002; 74: S1833-5; discussion S1857-63.
17) Erbel R, Alfonso F, Boileau C, et al. Diagnosis and management of aortic dissection. Eur Heart J 2001; 22: $1642-81$.

18) DeSanctis RW, Doroghazi RM, Austen WG, et al. Aortic dissection. N Engl J Med 1987; 317: 1060-7.

19) Suzuki S, Imoto K, Uchida K, et al. Midterm results of transluminal endovascular grafting in patients with DeBakey type III dissecting aortic aneurysms. Ann Thorac Cardiovasc Surg 2006; 12: 42-9

20) Steuer J, Eriksson MO, Nyman R, et al. Early and long-term outcome after thoracic endovascular aortic repair (TEVAR) for acute complicated type B aortic dissection. Eur J Vasc Endovasc Surg 2011; 41: 318-23

21) Svensson LG, Kouchoukos NT, Miller DC, et al. Expert consensus document on the treatment of descending thoracic aortic disease using endovascular stent-grafts. Ann Thorac Surg 2008; 85: S1-41.

22) Tang DG, Dake, MD. TEVAR for acute uncomplicated aortic dissection: Immediate repair versus medical therapy. Semin Vasc Surg 2009; 22: 145-51.

23) Nienaber CA, Kische S, Akin I, et al. Strategies for subacute/chronic type B aortic dissection: The Investigation Of Stent Grafts in Patients with type B Aortic Dissection (INSTEAD) trial 1-year outcome. J Thorac Cardiovasc Surg 2010; 140: S101-8; discussion S142-S146. 